\title{
Zika Virus Surveillance and Preparedness — New York City, 2015-2016
}

\author{
Christopher T. Lee, MD ${ }^{1,2}$; Neil M. Vora, MD 3,4; Waheed Bajwa, PhD ${ }^{5}$; Lorraine Boyd, MD ${ }^{6}$; Scott Harper, MD ${ }^{3,4}$; Daniel Kass, MSPH ${ }^{5}$; \\ Aileen Langston, $\mathrm{MD}^{6}$; Emily McGibbon, $\mathrm{MPH}^{3}$; Mario Merlino, MS, $\mathrm{MPH}^{5}$; Jennifer L. Rakeman, $\mathrm{PhD}^{7}$; Marisa Raphael, $\mathrm{MPH}^{8}$; \\ Sally Slavinski, DVM ${ }^{3}$; Anthony Tran, DrPH7; Ricky Wong9; Jay K. Varma, MD ${ }^{3,10}$; NYC Zika Response Team
}

\section{On June 21, 2016, this report was posted as an MMWR Early Release on the MMWR website (http://www.cdc.gov/mmwr).}

Zika virus has rapidly spread through the World Health Organization's Region of the Americas since being identified in Brazil in early 2015. Transmitted primarily through the bite of infected Aedes species mosquitoes, Zika virus infection during pregnancy can cause spontaneous abortion and birth defects, including microcephaly $(1,2)$. New York City (NYC) is home to a large number of persons who travel frequently to areas with active Zika virus transmission, including immigrants from these areas. In November 2015, the NYC Department of Health and Mental Hygiene (DOHMH) began developing and implementing plans for managing Zika virus and on February 1, 2016, activated its Incident Command System. During January 1-June 17, 2016, DOHMH coordinated diagnostic laboratory testing for 3,605 persons with travelassociated exposure, $182(5.0 \%)$ of whom had confirmed Zika virus infection. Twenty $(11.0 \%)$ confirmed patients were pregnant at the time of diagnosis. In addition, two cases of Zika virus-associated Guillain-Barré syndrome were diagnosed. DOHMH's response has focused on 1) identifying and diagnosing suspected cases; 2 ) educating the public and medical providers about Zika virus risks, transmission, and prevention strategies, particularly in areas with large populations of immigrants from areas with ongoing Zika virus transmission; 3) monitoring pregnant women with Zika virus infection and their fetuses and infants; 4) detecting local mosquito-borne transmission through both human and mosquito surveillance; and 5) modifying existing Culex mosquito control measures by targeting Aedes species of mosquitoes through the use of larvicides and adulticides.

\section{Current Testing and Epidemiologic Surveillance for Zika Virus}

Because commercial testing for Zika virus only recently became available, DOHMH coordinated diagnostic testing with health care providers and public health laboratories, particularly DOHMH's Public Health Laboratory and the New York State Department of Health Wadsworth Center. The testing process has varied with the evolution of CDC guidelines regarding whom should be tested and as local capacity for testing expanded. Initially, medical epidemiologists screened all health care provider requests for Zika virus testing (based on
CDC testing recommendations) for the presence of compatible symptoms and travel histories before authorizing testing (3).

On February 4, 2016, New York state testing criteria* were expanded to include asymptomatic pregnant women who traveled to an affected area at any time during pregnancy. This resulted in an increase in the number of patients for whom specimens were sent for reverse transcription-polymerase chain reaction (RT-PCR) and serology testing from a median of seven per day during January 21-February 3 to 52 per day during February 4-February 17 . As a result of the increased volume of requests, DOHMH withdrew the requirement for medical epidemiologist authorization on February 12 and began permitting providers to submit specimens directly to the Public Health Laboratory. However, because of subsequent receipt of a large number of specimens that were mislabeled, mishandled, or improperly processed; had incomplete or missing laboratory requisition forms; or were obtained from patients who did not meet testing criteria, the pre-authorization requirement was reinstituted on March 21.

To manage the increased volume of testing requests and ensure adequate specimen processing, DOHMH rapidly established a Zika Testing Call Center using personnel, equipment, software, and physical space that had been used for the NYC Ebola active monitoring program (4). The call center triages calls and approves testing requests from providers, completes and faxes laboratory requisition forms to providers to include with the specimen, and arranges, when necessary, transportation of specimens to the Public Health Laboratory via a commercial courier.

During January 1-June 17, 2016, DOHMH coordinated laboratory diagnostic testing for 3,605 persons at the Public Health Laboratory, Wadsworth Center, and CDC. Among all persons tested, 3,319 (92.1\%) had a Zika RT-PCR test, and 3,305 (91.7\%) had Zika serology testing, which included immunoglobulin M (IgM) antibody capture enzyme-linked immunosorbent assay (MAC-ELISA) and, for some patients, plaque reduction neutralization testing (PRNT). A total of 182 (5.0\%) confirmed cases of Zika virus infection were identified, based on positive results of urine or serum RT-PCR or serologic ${ }^{\dagger}$ testing. The majority of cases were confirmed by

\footnotetext{
*https://www.health.ny.gov/diseases/zika_virus/docs/2016-02-4_notification.pdf. $\dagger$ Positive result from IgM antibody capture enzyme linked immunosorbent assay (MAC-ELISA) with confirmatory plaque reduction neutralization test (PRNT).
} 
urine RT-PCR results (Table). Among all confirmed cases, 20 patients (11.0\%) were pregnant at the time of diagnosis, nine of whom had symptoms compatible with Zika virus disease. Two cases of Zika virus-associated Guillain-Barré syndrome were diagnosed. Based on PRNT, 27 additional patients $(0.7 \%$ of persons tested) were found to have unspecified recent flavivirus infection. All confirmed cases occurred in persons who had been in an area with ongoing Zika virus transmission.

To analyze possible undertesting based on residence, on March 1, DOHMH used U.S. Census American Community Survey, $2010-2014^{\S}$ data to map by census tract 1 ) the number of persons living in NYC who were born in Mexico, the Caribbean, Central America, or countries in South America with active transmission of Zika virus (Figure 1), because these persons might travel frequently to areas with active Zika virus transmission, and 2) Zika virus testing rates among women aged 15-44 years during January-February 2016 (Figure 2). This mapping found little correspondence between census tracts with high rates of Zika virus testing and census tracts with high numbers of immigrants from countries with ongoing Zika virus transmission. The highest testing rates among women aged 15-44 years (104 per 100,000 population) occurred in census tracts in the lowest quartile of immigrants from these countries; whereas, the lowest rates of testing (29 per 100,000) occurred in census tracts in the highest quartile of immigrants from countries with ongoing Zika virus transmission. To address this apparent demographic disparity in testing, DOHMH personnel distributed educational materials in English, Spanish, and 10 other languages to practices of 170 health care providers in areas with large immigrant populations. To educate the public, $\mathrm{DOHMH}$ responded to dozens of media inquiries, including 25 one-on-one interviews with Spanish language media; distributed approximately 10,000 Zika testing informational cards throughout the city and approximately 6,000 travel warning flyers for pregnant women; and conducted approximately 100 presentations at social, community, and religious gatherings throughout the city regarding prevention of mosquito bites. During April-May 2016, the testing rate among women aged $15-44$ years increased in census tracts with the highest quartile of immigrants $(65$ per 100,000) and decreased in census tracts with the lowest quartile of immigrants (40 per 100,000).

Pregnant women with confirmed Zika virus infection or inconclusive test results are followed for the duration of pregnancy by DOHMH medical epidemiologists in collaboration with their providers, and infants born to these women are periodically followed by DOHMH for the first 12 months of life. In mid-April, DOHMH convened a meeting with the City's nine Regional Perinatal Centers to review DOHMH interim

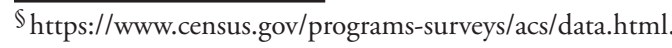

TABLE. Laboratory test results for 182 confirmed cases of Zika virus disease, by type of test - New York City, January 1-June 17, 2016

\begin{tabular}{lc}
\hline Laboratory test & $\begin{array}{c}\text { Confirmed cases } \\
\text { No. }(\%)\end{array}$ \\
\hline RT-PCR (total) & $178(97.8)$ \\
Urine and serum RT-PCR & $25(13.7)$ \\
Urine RT-PCR only & $117(64.3)$ \\
Serum RT-PCR only & $36(19.8)$ \\
Serology only* & $4(2.2)$ \\
\hline
\end{tabular}

Abbreviation: RT-PCR: reverse transcription-polymerase chain reaction.

* Positive result from immunoglobulin M antibody capture enzyme-linked immunosorbent assay (MAC-ELISA) with confirmatory plaque reduction neutralization test (PRNT).

guidance and solicit input on improving Zika preparedness and response in NYC.

\section{Surveillance to Detect Local Transmission}

During peak mosquito-biting season in NYC (JulySeptember), DOHMH will implement a sentinel surveillance system to detect human cases of local mosquito-borne transmission of Zika virus. DOHMH has selected 21 primary care clinics and emergency departments as sentinel sites across all five NYC boroughs, prioritizing areas with large populations of immigrants from countries with ongoing Zika virus transmission and areas where there have been previous travelassociated cases of other mosquito-borne infections, including chikungunya and dengue. A suspected case of locally acquired Zika virus disease will be defined as an illness including fever, maculopapular rash, and either arthralgia or conjunctivitis in a person aged $>5$ years with no history of travel to Zikaaffected areas during the preceding 4 weeks. Suspected cases will be reported to DOHMH, and urine will be obtained for RT-PCR testing. A confirmed Zika virus disease case from a sentinel site will trigger an epidemiologic investigation to rule out other sources of exposure (e.g., sexual transmission or blood donation) and confirm local transmission.

Additional methods for detecting local transmission include asking persons with confirmed and suspected cases if any household members who have not traveled reported similar illness, and relying on clinicians to recognize and report clusters of persons with Zika-like illness, but no travel to areas with known Zika virus transmission. Confirmation of vector-borne local transmission would also trigger an environmental response, including enhanced mosquito surveillance and directed mosquito source control strategies to eliminate mosquito breeding sites.

DOHMH expanded its existing West Nile virus mosquito surveillance program, which focuses on Culex species mosquitoes, to include Aedes sp. mosquitoes. DOHMH added 60 traps optimized for Aedes collection, thereby doubling the number of trap sites. Placement of the new traps is based on 
historical location of Aedes mosquitoes and natural and manmade environment features to enable interpolation of Aedes populations in areas without traps. Mosquitoes are collected, identified by species, and tested for Zika virus by RT-PCR at the Public Health Laboratory.

\section{Enhancement of Mosquito Control}

Because of the known potential for Aedes mosquitoes to transmit Zika virus among humans, the anticipated large number of imported human cases into NYC, and the temporal lag between viremia and disease diagnosis in an infected patient, DOHMH is augmenting its mosquito control program, specifically source control, as well as larviciding and adult mosquito control. Whereas the West Nile virus control program relies on location and population density of West Nile virus-infected Culexsp. mosquitoes to guide mosquito control, the Zika virus control program treats Aedes sp. mosquitoes as a public health hazard regardless of infection status, with the application of larvicides and adulticides calibrated to mosquito surveillance data.

Aerial application of larvicide over unpopulated marshland and freshwater wetlands began during the week of May 9. Application of larvicide to catch basins will occur four times during mosquito season. As Aedes sp. mosquitoes reach a significant number (average of 25 mosquitoes per trap-day, subject to reconsideration based on surveillance findings), $\mathrm{DOHMH}$ will conduct truck-based, ultra-low volume spraying of biorational larvicide and chemical adulticide in residential areas. Inspectors from DOHMH will identify potential mosquito breeding sites in the city and work closely with communities to increase awareness of the need to eliminate pools of standing water in residential areas.

\section{Discussion}

In the United States, Zika virus disease cases have occurred after travel to affected areas and through sexual transmission $(5,6)$. Areas with imported cases of Zika virus disease and local circulation of Aedes sp. mosquitoes are at increased risk for local mosquito-borne transmission of Zika virus. Although Aedes aegypti mosquitoes have never been documented in NYC, CDC estimates a potential range north of NYC, and a related species, Ae. albopictus is present in NYC and is a potentially competent Zika virus vector ( 7 ).

Activation of the Incident Command System in NYC allowed rapid mobilization of 328 pre-designated DOHMH

\footnotetext{
Biorational pesticides are distinguished from conventional pesticides by the Environmental Protection Agency as those with a nontoxic mode of action, low use volume, target species specificity, and natural occurrence. Additional information is available at http://nepis.epa.gov/Exe/ZyPDF.cgi/9101DPEI. PDF?Dockey=9101DPEI.PDF.
}

\section{Summary \\ What is already known about this topic?}

Zika virus emerged in the Region of the Americas in early 2015, and imported cases have been detected in the United States, including New York City (NYC).

What is added by this report?

As of June 17, 2016, a total of 3,605 patients had been tested for Zika virus in NYC, 182 (5.0\%) of which have been confirmed cases of Zika infection; 20 cases were in women who were pregnant at the time of diagnosis, and two cases of GuillainBarré syndrome were diagnosed. The majority of cases were diagnosed by urine reverse transcription-polymerase chain reaction. The presence of a potentially competent Aedes mosquito vector in NYC necessitates a health department-wide response to identify and respond to potential local transmission of Zika virus, including sentinel surveillance and enhanced mosquito control.

What are the implications for public health practice?

In NYC, pregnant women and persons with a Zika-like illness who have been in Zika virus-affected areas should be tested for Zika virus infection. Providers should offer up-to-date information on the risk for birth defects so that pregnant patients can make informed decisions about pregnancy options. Preparedness for local transmission of Zika virus involves a robust emergency response infrastructure, targeted public health messaging, human and environmental surveillance strategies, and an integrated epidemiologic, clinical, and environmental response.

personnel to enhance human and mosquito surveillance, public and provider awareness, and vector control. All DOHMH employees have emergency response roles officially included in their job descriptions as a mandatory condition of employment, and they undergo training at regular intervals for specific Incident Command System roles regardless of the type of emergency. DOHMH's Zika response relied upon emergency capacities first developed in 1999 in response to West Nile virus and notably expanded in response to the 2014-2015 Ebola emergency, which involved epidemiologists, microbiologists, community outreach workers, emergency preparedness specialists, and equipment and supplies supported by city, state, and federal funds.

DOHMH has worked directly with the public and health care providers to increase awareness about Zika virus risks, prevention strategies, and testing recommendations. Health care providers should offer up-to-date information on the risk for birth defects so that pregnant patients can make informed decisions about pregnancy options. DOHMH has contributed substantial resources to increasing access to testing for persons at risk for Zika virus infection by establishing a Zika Testing Call Center and using geospatial analysis to identify neighborhoods with possible undertesting, based on 
FIGURE 1. Number of persons born in Mexico, the Caribbean, Central America, and countries in South America with active Zika virus transmission, by U.S. Census tract of residence - New York City, January-February 2016

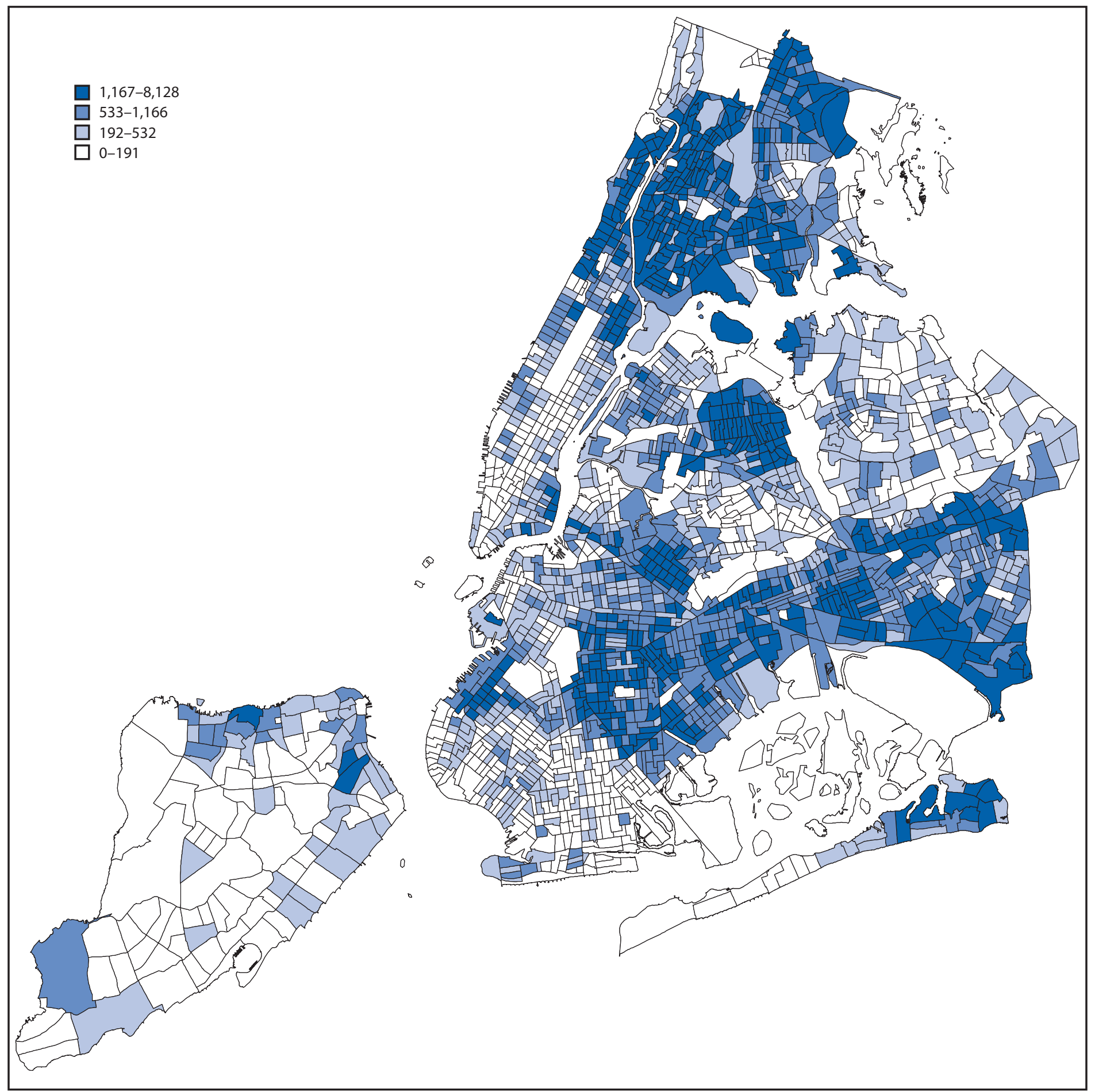


FIGURE 2. Zika virus testing rate per 10,000 among females aged 15-44 years, by U.S. Census tract of residence — New York City, JanuaryFebruary 2016

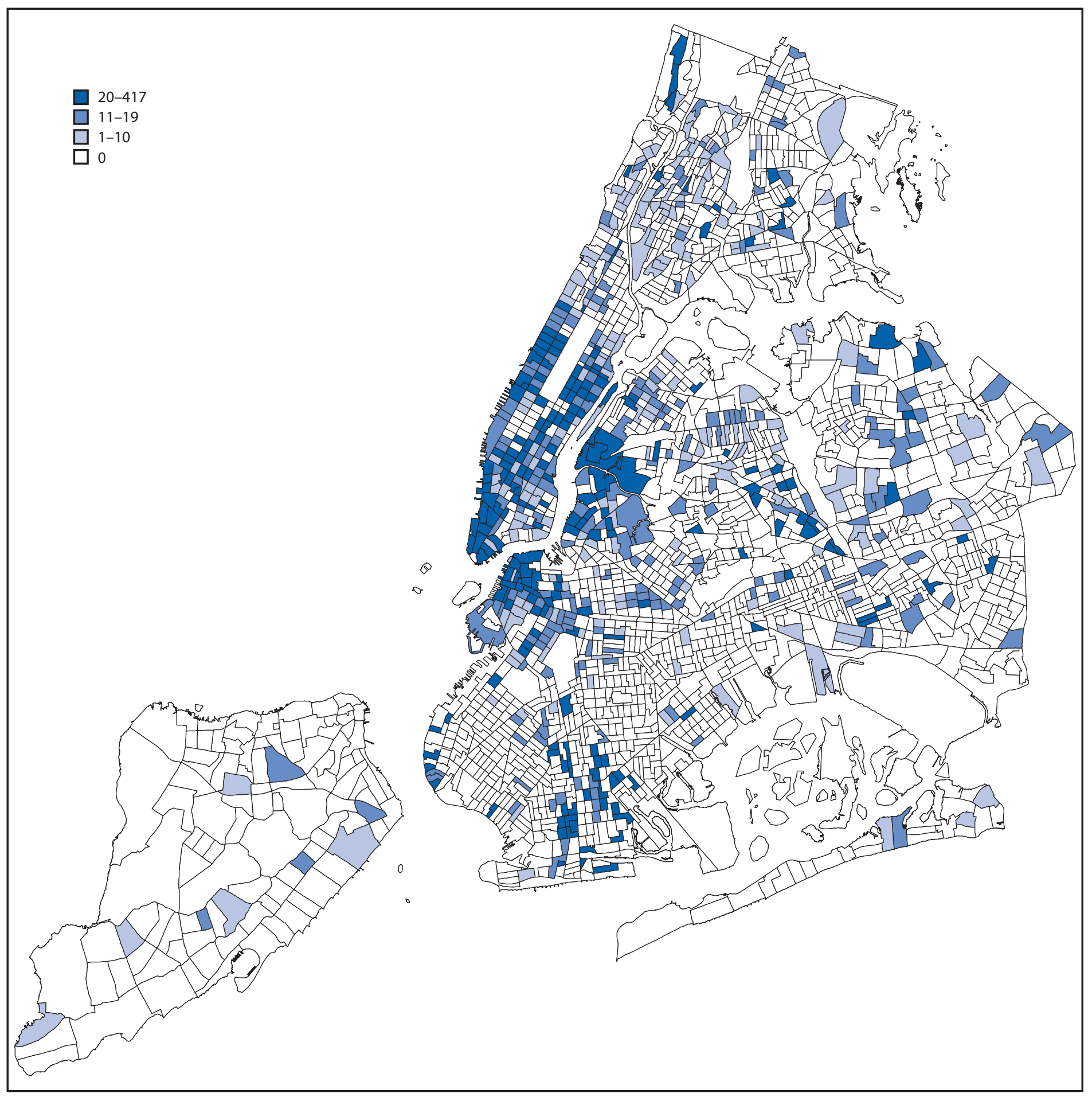


demographic population characteristics. NYC has invested substantially in expanding Zika virus testing capacity, allowing the Public Health Laboratory to receive and process several hundred specimens each day; however, capacity for testing might be insufficient to meet demand if sustained Zika virus transmission in the United States occurs. In the event of local mosquito-borne transmission, public health laboratory resources might need to prioritize testing among certain groups, including pregnant women and patients with Guillain-Barré syndrome. In the event that testing demand exceeds capacity and NYC DOHMH is unable to return test results within 2 weeks, NYC will collaborate with Wadsworth Center and CDC to facilitate testing. Public health agency collaboration with external partners might expedite availability of clinical nucleic acid and serologic testing at commercial clinical labs.

With the exception of suspected cases of sexually transmitted or congenital Zika virus infection, testing in NYC is currently limited to persons who have been to an area with ongoing Zika virus transmission, which precludes detection of cases acquired from a local mosquito bite. Implementation by DOHMH of sentinel surveillance for Zika virus infection in persons with a clinically compatible illness and no history of travel to an area with ongoing Zika virus transmission will facilitate rapid identification of locally transmitted Zika virus disease. Despite the absence of local mosquito-borne transmission or Zika virus-infected mosquitoes currently, expanded source control and applied larvicides and adulticides for Aedes mosquitoes might reduce the likelihood for local transmission. Similar approaches could be considered in other jurisdictions that are likely to have large numbers of imported human cases of Zika virus disease and potential Zika vectors.

\footnotetext{
${ }^{1}$ Division of Epidemiology, New York City Department of Health and Mental Hygiene; ${ }^{2}$ Epidemic Intelligence Service, CDC; ${ }^{3}$ Division of Disease Control, New York City Department of Health and Mental Hygiene; ${ }^{4}$ Office of Public Health Preparedness and Response, CDC; ${ }^{5}$ Division of Environmental Health, New York City Department of Health and Mental Hygiene; ${ }^{6}$ Division of Family and Child Health, New York City Department of Health and Mental Hygiene; ${ }^{7}$ Public Health Laboratory, New York City Department of Health and Mental Hygiene; ${ }^{8}$ Office of Emergency Preparedness and Response, New York City Department of Health and Mental Hygiene; ${ }^{9}$ Office of External Affairs, New York City Department of Health and Mental Hygiene; ${ }^{10}$ National Center for Emerging and Zoonotic Infectious Diseases, CDC.

Corresponding author: Christopher Lee, klq5@cdc.gov, 347-396-4070.
}

\section{NYC Zika Response Team}

Bisrat Abraham, MD, Division of Disease Control, New York City Department of Health and Mental Hygiene; Joel Ackelsberg, MD, Division of Disease Control, New York City Department of Health and Mental Hygiene; Mike Antwi, MD, Division of Disease Control, New York City Department of Health and Mental Hygiene; Sharon Balter, MD, Division of Disease Control, New York City Department of and Mental Hygiene; Jennifer Baumgartner, MSPH, Division of Disease Control, New York City Department of Health and Mental Hygiene; Isaac Benowitz, MD, Division of Disease Control, New York City Department of Health and Mental Hygiene, Epidemic Intelligence Service, CDC; Marie Antoinette Bernard, MD, Division of Disease Control, New York City Department of Health and Mental Hygiene; Angelica Bocour, MPH, Division of Disease Control, New York City Department of Health and Mental Hygiene; Christine Borges, MPH, Division of Disease Control, New York City Department of Health and Mental Hygiene; Joseph Burzynski, MD, Division of Disease Control, New York City Department of Health and Mental Hygiene; James E. Cone, MD, Division of Epidemiology, New York City Department of Health and Mental Hygiene; Alexander Davidson, MPH, Division of Disease Control, New York City Department of Health and Mental Hygiene; Paula Del Rosso, Division of Disease Control, New York City Department of Health and Mental Hygiene; Catherine Dentinger, FNP, Division of Disease Control, New York City Department of Health and Mental Hygiene, Office of Public Health Preparedness and Response, CDC; Bisram Deocharan, PhD, Division of Disease Control, New York City Department of Health and Mental Hygiene; Marie Dorsinville, MPH, Division of Disease Control, New York City Department of Health and Mental Hygiene; Peter Ephross, MA, Division of Epidemiology, New York City Department of Health and Mental Hygiene; Ifeoma Ezeoke, MPH, Division of Disease Control, New York City Department of Health and Mental Hygiene; Anne Fine, MPH, Division of Disease Control, New York City Department of Health and Mental Hygiene; Ana Maria Fireteanu, MPH, Division of Disease Control, New York City Department of Health and Mental Hygiene; Jie Fu, PhD, Division of Disease Control, New York City Department of Health and Mental Hygiene; Sharon K. Greene, PhD, Division of Disease Control, New York City Department of Health and Mental Hygiene; Scott Hughes, PhD, Division of Disease Control, New York City Department of Health and Mental Hygiene; Maryam Iqbal, MS, Division of Disease Control, New York City Department of Health and Mental Hygiene; Jasmine Jacobs-Wingo, MPH, Office of Emergency Public Health Preparedness and Response, New York City Department of Health and Mental Hygiene, Office of Public Health Preparedness and Response, CDC; Kimberly Johnson, MS, Division of Environmental Health, New York City Department of Health and Mental Hygiene; Lucretia Jones, DrPH, Division of Disease Control, New York City Department of Health and Mental Hygiene; Hannah T. Jordan, MD, Division of Epidemiology, New York City Department of Health and Mental Hygiene; Fabienne Laraque, MD, Division of Disease Control, New York City Department of Health and Mental Hygiene; Marcelle Layton, MD, Division of Disease Control, New York City Department of Health and Mental Hygiene; David Lee, MPH, MBA, Division of Disease Control, New York City Department of Health and Mental Hygiene; Ellen Lee, MD, Division of Disease Control, New York City Department of Health and Mental Hygiene; Kristen Lee, MPH, Division of Disease Control, New York City Department of Health and Mental Hygiene; Dakai Liu, PhD, Division of Disease Control, New York City Department of Health and Mental Hygiene; David E. Lucero, PhD, Division of Disease Control, New York City Department of Health and Mental 
Hygiene; Michelle Macaraig, DrPH, Division of Disease Control, New York City Department of Health and Mental Hygiene; Natasha McIntosh, Division of Disease Control, New York City Department of Health and Mental Hygiene; Paul McNamee, MPH, Division of Disease Control, New York City Department of Health and Mental Hygiene; Julie Myers, MD, Division of Disease Control, New York City Department of Health and Mental Hygiene; Stephanie Ngai, MPH, Division of Disease Control, New York City Department of Health and Mental Hygiene; Pete Papadopolous, MPH, Division of Disease Control, New York City Department of Health and Mental Hygiene; Hilary Parton, MPH, Division of Disease Control, New York City Department of Health and Mental Hygiene; Eric Peterson, $\mathrm{MPH}$, Division of Disease Control, New York City Department of Health and Mental Hygiene; Carolina Pichardo, Division of Disease Control, New York City Department of Health and Mental Hygiene; Robert Pirillo, Division of Disease Control, New York City Department of Health and Mental Hygiene; Celia Quinn, MD, Office of Emergency Preparedness and Response, New York City Department of Health and Mental Hygiene, Office of Public Health Preparedness and Response, CDC; Vasudha Reddy, MPH, Division of Disease Control, New York City Department of Health and Mental Hygiene; Alhaji Saffa, MPH, Division of Disease Control, New York City Department of Health and Mental Hygiene; Altaf Shaikh, MS, Division of Informatics and Information Technology, New York City Department of Health and Mental Hygiene; Alaina Stoute, MPH, Division of Disease Control, New York City Department of Health and Mental Hygiene; Anna Tate, MPH, Division of Disease Control, New York City Department of Health and Mental Hygiene; Brian Toro, Division of Disease Control, New York City Department of Health and Mental Hygiene; Olivia C. Tran, MPH, Division of Disease Control, New York City Department of Health and Mental Hygiene; Elaine Vernetti, MD, Office of Emergency Preparedness and Response, New York City Department of Health and Mental Hygiene; Don Weiss, MD, Division of Disease Control, New York City Department of Health and Mental Hygiene; Emily Westheimer, MSc, Division of Disease Control, New York City Department of Health and Mental Hygiene; Eliza Wilson, Division of Disease
Control, New York City Department of Health and Mental Hygiene; Ann Winters, MD, Division of Disease Control, New York City Department of Health and Mental Hygiene; Marie Wong, Division of Disease Control, New York City Department of Health and Mental Hygiene; Winfred Y. Wu, MD, Division of Prevention and Primary Care, New York City Department of Health and Mental Hygiene; David Yang, Division of Disease Control, New York City Department of Health and Mental Hygiene; Mohammad Younis, MPA, Division of Disease Control, New York City Department of Health and Mental Hygiene; Janette Yung, MPH, Division of Disease Control, New York City Department of Health and Mental Hygiene.

\section{References}

1. Pan American Health Organization. Epidemiological alert: neurological syndrome, congenital malformations, and Zika virus infection. Implications for public health in the Americas. Washington, DC: World Health Organization, Pan American Health Organization; 2015. http://www.paho.org/hq/index. php?option=com_docman\&task=doc_download \&Itemid=\&gid $=32405$

2. Rasmussen SA, Jamieson DJ, Honein MA, Petersen LR. Zika virus and birth defects-reviewing the evidence for causality. N Engl J Med 2016;374:1981-7. http://dx.doi.org/10.1056/NEJMsr1604338

3. Petersen EE, Staples JE, Meaney-Delman D, et al. Interim guidelines for pregnant women during a Zika virus outbreak-United States, 2016. MMWR Morb Mortal Wkly Rep 2016;65:30-3. http://dx.doi. org/10.15585/mmwr.mm6502e1

4. Millman AJ, Chamany S, Guthartz S, et al. Active monitoring of travelers arriving from Ebola-affected countries-New York City, October 2014April 2015. MMWR Morb Mortal Wkly Rep 2016;65:51-4. http:// dx.doi.org/10.15585/mmwr.mm6503a3

5. Armstrong P, Hennessey M, Adams M, et al. Travel-associated Zika virus disease cases among U.S. residents-United States, January 2015February 2016. MMWR Morb Mortal Wkly Rep 2016;65:286-9. http:// dx.doi.org/10.15585/mmwr.mm6511e1

6. Oster AM, Russell K, Stryker JE, et al. Update: interim guidance for prevention of sexual transmission of Zika virus-United States, 2016 MMWR Morb Mortal Wkly Rep 2016;65:323-5. http://dx.doi. org/10.15585/mmwr.mm6512e3

7. Wong PS, Li MZ, Chong CS, Ng LC, Tan CH. Aedes (Stegomyia) albopictus (Skuse): a potential vector of Zika virus in Singapore. PLoS Negl Trop Dis 2013;7:e2348. http://dx.doi.org/10.1371/journal.pntd.0002348 